\title{
Sonographic features and management options of uterine arteriovenous malformation. Six cases report.
}

\author{
Halil Aslan', Deniz Kanber Acar', Ali Ekiz', Basak Kaya1, Salim Sezer', Reshad Ismayilzade², \\ Murat Can'
}

${ }^{1}$ Department of Maternal Fetal Medicine, ${ }^{2}$ Department of Obstetrics and Gynecology, Kanuni Sultan Suleyman Education and Research Hospital, Istanbul, Turkey.

\begin{abstract}
Uterine arteriovenous malformation (AVM), an extremely rare condition, is defined as an abnormal connection between artery and vein. Although the pelvis is a common site for AVM, the uterus is involved occasionally and the true incidence is unknown. The objective of this study was to discuss diagnostic features and management options of AVMs. In this paper six cases of AVMs have been reported with a review of diagnosis and management options.

Keywords: uterus, arteriovenous malformation, Doppler ultrasound, angiography, embolization
\end{abstract}

Abstract

\section{Introduction}

Uterine arteriovenous malformation (AVM) is a rare but potentially life-threatening condition. It is a vascular lesion formed by the abnormal connection between artery and vein. Although the pelvis is a common site for AVM, the uterus is occasionally involved [1] and the true incidence is unknown [2]. The most common clinical manifestation is recurrent vaginal bleeding or menorrhagia [3]. The other presentations are postpartum hemorrhage, postmenopausal bleeding, infertility, spontaneous abortion, and congestive heart failure [4,5]. Color Doppler ultrasonography (CDUS) has become the preferred diagnostic method in the last two decades. However, angiography remains the gold-standard modality for diagnosing uterine AVM, as it is required for both definitive diagnosis and treatment.

In this paper six cases of AVMs have been reported with a review of diagnosis and management strategies.

Received 09.12.2014 Accepted 21.01.2015

Med Ultrason

2015, Vol. 17, No 4, 561-563

Corresponding author: Ali Ekiz M.D.

Fevzi Cakmak mah. Barbaros cd. 775. Sk

Validesuyu Konutlari C2 blok D:34

Gaziosmanpasa, Istanbul/Turkey

Phone: +90 505962 6936, Fax: +90 2125714790

E-mail:draekiz@gmail.com

\section{Case 1}

A 22-year-old woman, Gravida 2 Para 1, presented to the emergency room with heavy vaginal bleeding and passage of blood clots. One month earlier, a cesarean delivery had been complicated by a hematoma measuring $5 \mathrm{~cm}$ at the right corner of the uterine incision, which required a repeat laparotomy because of a postoperative abscess (day 21). On admission vaginal examination findings were normal. Transvaginal ultrasonography (TVUS) showed a $3 \mathrm{~cm}$ vascular mass with a turbulent intraluminal flow. CDUS suggested the diagnosis of uterine artery pseudo aneurysm (fig 1). A pelvic angiography was planned. But a sudden massive vaginal bleeding appeared and the hemoglobin dropped from 10.9 to $6.0 \mathrm{~g} / \mathrm{dl}$. General measures including

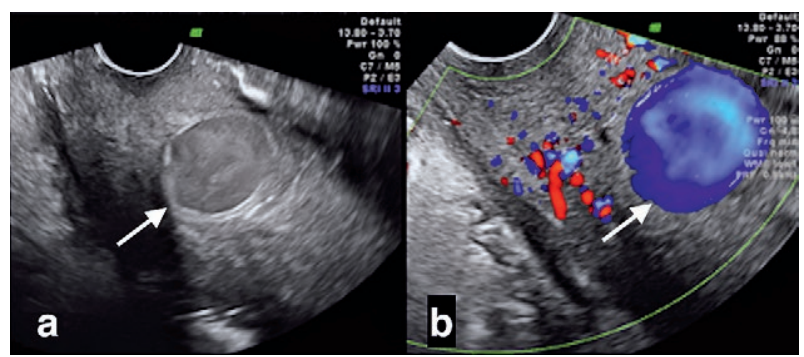

Fig 1. a) Gray-scale ultrasonography: the arrow shows the centrally located AVM with a turbulent flow; b) the turbulent flow in AVM was evidenced via Color Doppler ultrasonography. 
blood transfusion and intrauterine Foley catheter application could not control the bleeding. As a life-saving procedure, a hysterectomy was performed. Postoperative period was uneventful and the patient was discharged on Day 5. Except for extensive inflammation and areas of hemorrhage, pathologic findings were unremarkable.

\section{Case 2}

A 27-year-old woman, Gravida 2, Para 1, abort 1, presented to the emergency room with severe vaginal bleeding. She had had a spontaneous abortion and a uterine curettage 2 months before in an outpatient clinic. Over the previous week she reported vaginal bleeding which stopped spontaneously. Physical examination revealed a pale woman with a blood pressure of 90/50 $\mathrm{mmHg}$ and a pulse rate of 120 beat per minute. There were blood clots in the vagina and active vaginal bleeding from a normalappearing cervical os. Laboratory evaluation revealed profound anemia with a hemoglobin count of $4.7 \mathrm{~g} / \mathrm{dl}$ and a normal platelet count of 272000 . Serum $\beta$ hCG level was $<2 \mathrm{mIU} / \mathrm{ml}$. Except for a mildly enlarged uterus, initial transvaginal TVUS findings were unremarkable.

The patient was transfused with 3 units of packed red cell. After placement of an intrauterine Foley catheter, vaginal bleeding subsided. A repeat TVUS revealed a distended uterine cavity in which there was a hyperechogenic mass surrounded by an ananechoic zone. CDUS examination evidenced slowly moving blood without pulsation in the uterine cavity suggesting the diagnosis of a direct arterial branch rupture. A pelvic angiogram confirmed the diagnosis (fig 2). Uterine arterial embolization was performed and the patient was discharged on the third day of hospitalization.

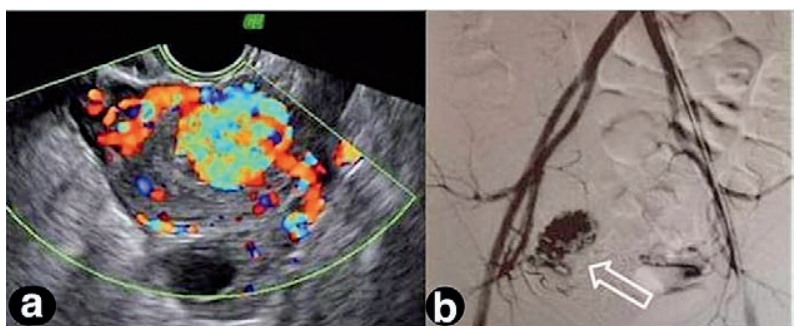

Fig 2. The mosaic pattern of color signals within the cystic spaces was demonstrated with Color Doppler ultrasonography (a). Angiogram evidenced a marked visualized vascular tangle (arrow) (b).

\section{Cases 3-6}

Cases 3, 4, 5 and 6 were similar; the patients were referred for severe vaginal bleeding. In all four cases TVUS revealed multiple anechoic, tortuous spaces in the uterine walls extending to the uterine cavity and CDUS showed a mosaic pattern caused by multidirectional, turbulent flow consistent with an arteriovenous flow pattern suggesting a uterine AVM. Pelvic angiograms confirmed the diagnosis and uterine arteries were successfully embolized. We also documented an episode of profuse vaginal bleeding at the time of examination by transvaginal B-mode and CDUS (fig 3).

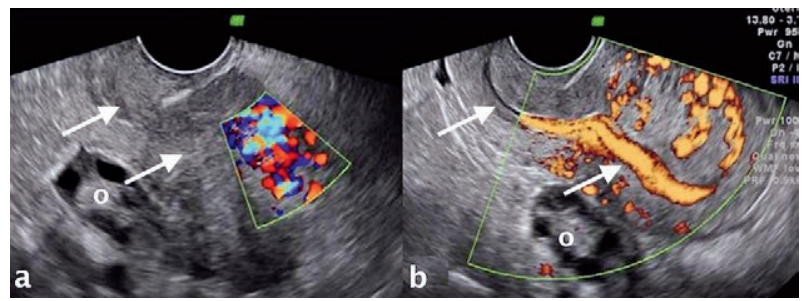

Fig 3. The patient had profuse bleeding at the time of examination: a) longitudinal scan, (Color Doppler) of the uterus showed a mosaic pattern of color signals in the anterior wall. Arrows also showed no collection in the uterine cavity; b) after profuse bleeding had started, unique findings were recorded including; the two layer of the endometrial wall were separated (arrows), power Doppler ultrasonography demonstrated bleeding flow from the uterine fundus to cervical canal (arrows). O: Ovary

\section{Discussions}

A wide spectrum of uterine vascular abnormalities has been defined and classified as uterine artery aneurysm, uterine artery pseudoaneurysms, arteriovenous malformations, and direct arterial branch ruptures. All of these are rare abnormalities, which can be asymptomatic or present with intractable recurrent heavy vaginal bleeding in women of any age. Although, AVMs can be congenital or acquired, commonly occurrences follow a previous uterine trauma due to surgery, delivery, abortion, dilatation and curettage or infection [6].

The diagnosis of AVM is troublesome because it is a rare malformation and unable to be diagnosed in a routine examination. Gray-scale ultrasonography is the most commonly performed initial imaging examination for uterine hemorrhage [7]. Even though the sonographic features are nonspecific, it could alert the examiner to the presence of a vascular abnormality. These include an ill-defined uterine mass consisting of mildly echogenic tissue that is interspersed with multiple hypoechogenic spaces of varying sizes [8]. Color Doppler improves the diagnostic ability of sonography [9]. The typical finding on Color Doppler ultrasonography is a tangle of tortuous vessels with a multidirectional, high-velocity and turbulent flow. Although contrast-enhanced computed tomography $(\mathrm{CT})$ and magnetic resonance imaging (MRI) can be used, conventional angiography is the goldstandard technique in diagnosis $[10,11]$. 
Differential diagnosis includes retained products of conception, hemangiomas, varicosities, uterine sarcoma, and trophoblastic disease [8]. B-hCG level should require distinguishing uterine AVM from invasive mole or choriocarcinoma.

Acute treatment consists of hemodynamic stabilization including blood transfusion and utero-cervico-vaginal tamponade [7]. Uterine AVM has been traditionally managed by a hysterectomy. For patients who desire future fertility, uterine artery embolization is performed with a success rate $>95 \%$ in obstetric and gynecologic bleeding [12]. The advantages of embolization include minimal invasion with less blood loss, shorter hospital stay, faster recovery, and the preservation of uterus [7].

Alternative uterus preserving procedures are isolation of AVM, unilateral uterine/ovarian artery ligation, laparoscopic bipolar coagulation of uterine vessels and bilateral hypogastric artery ligation [7]. Gonadotropin-releasing hormone, methylergonovine maleate, and danazol have been also used in a few cases [13]. Timmerman et al have described regression of the AVM with conservative management or spontaneous resolution [14,15]. Additionally, Lee et al have concluded that ultrasonography can accurately predict selection of conservative management [16]. Although there are a few reports suggesting spontaneous resolution of AVMs in the literature, we have not experienced spontaneous resolution of AVM among the six patients.

In our series one patient was given an urgent hysterectomy as a life saving procedure and the remaining five patients were successfully treated with an embolization procedure. With regard to our data, embolization is a highly effective method in treating uterine AVM. Concordantly, Wang et al reported an $88 \%$ overall clinical success rate of embolization [17]. Of interest, we also noticed that all patients were relatively young and had a high cesarean section ratio. From this point of view, the increasing number of cesarean sections in the worldwide population probably has an important effect on the increasing number of uterine vascular malformations. To the best of our knowledge, this is the first case report of uterine AVM, which started to bleed at the time of examination. The Doppler ultrasonography findings of acute and profuse bleeding were demonstrated in this paper.

In conclusion, uterine AVMs are rare but a potentially life-threatening condition and are much more common than previously thought. Uterine AVM should be kept in mind if there is an unexpected, excessive, intermittent vaginal bleeding, particularly after delivery or after surgical procedures to the uterus. Further investigations should be performed starting with B-Mode and Color Doppler ultrasound to determine which patients need further invasive procedure.

\section{References}

1. Valenzano M, Foglia G, Tedeschi C, Paoletti R, Fulcheri E. Color Doppler sonography of uterine arteriovenous malformation. J Clin Ultrasound 2000; 28: 146-149.

2. Vijayakumar A, Srinivas A, Chandrashekar BM, Vijayakumar A. Uterine vascular lesions. Rev Obstet Gynecol 2013; 6: 69-79.

3. Polat P, Suma S, Kantarci M, Alper F, Levent A. Color Doppler US in the evaluation of uterine vascular abnormalities. Radiographics 2002; 22: 47-53.

4. Mohamed SI, Abdullah BJJ, Omar SZ. Postpartum hemorrhage. Biomed Imaging Interv J 2005; 1: e13. doi: 10.2349/ biij.1.2.e15.

5. Dighe M, Cuevas C, Moshiri M, Dubinsky T, Dogra VS. Sonography in first trimester bleeding. J Clin Ultrasound 2008; 36: 352-366.

6. Yang JJ, Xiang Y, Wan XR, Yang XY. Diagnosis and management of uterine arteriovenous fistulas with massive vaginal bleeding. Int J Gynaecol Obstet 2005; 89: 114-119.

7. Patel S, Potti S, Jaspan D, Dandolu V. Embolization of uterine arteriovenous malformation for treatment of menorrhagia. Arch Obstet Gynecol 2009; 279: 229-232.

8. Mungen E. Vascular abnormalities of the uterus: Have we recently over-diagnosed them? Ultrasound Obstet Gynecol 2003; 21: 529-531.

9. Kelly SM, Belli AM, Campbell S. Arteriovenous malformation of the uterus associated with secondary postpartum hemorrhage. Ultrasound Obstet Gynecol 2003; 21: 602605.

10. Pan HA, Kuo PL. Transvaginal color Doppler ultrasonography for detecting acquired arteriovenous malformation of the uterus. Taiwan J Obstet Gynecol 2002; 14: 25-29.

11. Cura M, Martinez N, Cura A, Dalsaso TJ, Elmerhi F. Arteriovenous malformations of the uterus. Acta Radiol 2009; 50: 823-829.

12. Wald DA. Postpartum hemorrhage resulting from uterine artery pseudoaneurysm. J Emerg Med 2003; 25: 57-60.

13. Morikawa M, Yamada T, Yamada H, Minakami H. Effect of gonadotropin-releasing hormone agonist on a uterine arteriovenous malformation. Obstet Gynecol 2006; 108: 751-753.

14. Timmerman D, Wauters J, Van Calenbergh S, et al. Color Doppler imaging is a valuable tool for the diagno- sis and management of uterine vascular malformations. Ultrasound Obstet Gynecol 2003; 21: 570-577.

15. Timmerman D, Van der Bosch T, Peeraer K, et al. Vascular maformations in the uterus; ultrasonographic diagnosis and conservative management. Eur J Obstet Gynecol Reprod Biol 2000; 92: 171-178.

16. Lee TY, Kim SH, Lee HJ, et al. Ultrasonographic indications for conservative treatment in pregnancy-related uterine arteriovenous malformations. Acta Radiol. 2014; 55): 1145-1152.

17. Wang Z, Chen J, Shi H, et al. Efficacy and safety of embolization in iatrogenic traumatic uterine vascular malformations. Clin Radiol 2012; 67: 541-545. 